\title{
Prediction of risk of depressive symptoms in menopausal women based on hot flash and sweating symptoms: a multicentre study
}

This article was published in the following Dove Press journal:

Clinical Interventions in Aging

Number of times this article has been viewed

\author{
Yanwei Zheng' \\ Yibei Zhou' \\ Jiangshan $\mathrm{Hu}^{\prime}$ \\ Jieping Zhu ${ }^{2}$ \\ Qi Hua ${ }^{3}$ \\ Minfang Tao' \\ 'Department of Gynecology and \\ Obstetrics, Shanghai Jiao Tong \\ University Affiliated Sixth People's \\ Hospital, ${ }^{2}$ Department of Gynecology \\ and Obstetrics, Shanghai Sixth \\ People's Hospital East Branch, \\ ${ }^{3}$ Department of Gynecology and \\ Obstetrics, International Peace \\ Maternal and Child Health Hospital of \\ the China Welfare Institute, Shanghai, \\ People's Republic of China
}

Objective: The present study aimed to develop a symptom-based (namely, hot flashes and sweating) scoring system for predicting the risk of depressive symptoms in menopausal women via a multicentre cross-sectional survey.

Methods: The data examined in the present study were obtained from 1,004 women aged 40-60 years who underwent physical examination at A Hospital. The basic information was obtained using a questionnaire-based survey. A self-rating depression scale was used to obtain the depressive symptom scores, while the Kupperman Menopausal Index was used to obtain the scores for the frequency of hot flashes and sweating. A logistic regression model was also established. The resulting $\beta$ coefficient was employed to calculate and predict the risk of depressive symptoms in these women and a risk scoring system was established. The scoring system was validated using samples from 2 other centers (validation sample 1: B Hospital, 440 women; validation sample 2: C Hospital, 247 women).

Results: The scoring system developed to predict the risk of depressive symptoms in menopausal women was based on hot flash and sweating symptoms and associated with menopausal status, hot flash scores, education level (high school education and below) and being diabetic. The scoring system yielded a total score of $0-54$ points. For women in the study sample, the area under the curve (AUC) of depressive symptom risk score was 0.750 (95\% CI, 0.708-0.793). Validation sample 1 had an AUC of 0.731 (95\% CI, 0.667-0.794), while validation sample 2 had an AUC of 0.744 (95\% CI, 0.669-0.820). The optimal cut-off score to assess depressive symptoms in women participating in the present study was 31 points. The sensitivity and specificity for predicting depressive symptoms in the study sample were 0.667 and 0.701 , respectively. In contrast, the sensitivity was 0.840 in validation sample 1 and 0.879 in validation sample 2 .

Conclusion: The hot flash and sweating symptom-based scoring system developed to predict the risk of depressive symptoms in menopausal women relies on non-laboratory survey data. The system is simple, practical, and convenient to use. For Chinese huge population of menopausal women, the scoring system should be considered a reliable screening tool for depressive symptoms.

Keywords: menopause, depressive symptoms, hot flash, sweating

\section{Introduction}

Depression is a major cause of disability in women. Approximately one-fifth of women are affected by depression, which is twice the rate of depression in men; however, the underlying reason remains unclear. ${ }^{1}$ The prevalence of depressive symptoms in menopausal women is $\sim 5.9 \%-23.8 \% .^{2-4}$ Studies have shown that the risk of experiencing depressive mood is 2 - to 3 -fold higher in perimenopausal women than in premenopausal 
women..$^{5-7}$ Estimates indicate that $\sim 73$ million adult women worldwide suffer from depressive disorder each year. ${ }^{8}$

Although depressive symptoms do not always equal depressive disorder, they represent an adverse effect of depressive disorder. In addition, depressive symptoms may enhance the risk of developing cardiovascular diseases, diabetes, metabolic syndrome, and chronic pain. Depressive symptoms have become a significant health burden. ${ }^{7,9}$

Most women reach menopause between the ages of 40 and 60 years. For Chinese women and women from Western countries, the average age at menopause is $\sim 50$ years. ${ }^{10,11}$ Vasomotor symptoms are one of the major health problems plaguing most menopausal women. However, symptom severity, frequency, and duration vary greatly due to individual differences. Literature reports indicate that nearly $80 \%$ of women experience hot flashes and sweating during the peri- or postmenopausal stage. ${ }^{12,13}$ Approximately 55\% of women develop hot flashes and sweating before the occurrence significant changes in the menstrual cycle occur. ${ }^{14}$

The Study of Women's Health Across the Nation (SWAN) reported that perimenopause may be a risk factor affecting depressive symptoms in women. ${ }^{15}$ The vasomotor symptoms that most commonly occur during the perimenopausal stage, such as hot flashes and sweating, may also contribute to the development of depressive symptoms in women. ${ }^{16-18}$ At present, screening for depressive symptoms in menopausal women mainly relies on scoring scales. However, this approach is time-consuming and requires assistance from professionals or trained personnel. In addition, the assessment results are subjectively influenced by the evaluators. Therefore, such an approach has a very limited application in large-scale screening.

A risk score is based on demographic statistics and basic anthropometric measurements. No laboratory tests and complex scale assessments are involved. As a practical and simple tool, the risk score is an effective evaluation strategy for many diseases. ${ }^{19,20}$ This approach is very practical for predicting depressive symptoms in China's growing population of menopausal women. The aim of the present study was to develop a risk assessment model based on hot flashes and sweating symptoms. Such a model should be able to predict the risk of developing depressive symptoms in menopausal women. In addition, it should meet clinicians' needs for simplicity and be suitable for use in large-scale screening efforts.

\section{Methods}

\section{Study setting and participants}

This was a multicenter cross-sectional study. The included data were collected from women who underwent physical examinations between February 01, 2016 and August 01, 2016 at the Health Examination Center of A, B, and C Hospitals. Hospital A, B, and C are Shanghai Jiao Tong University Affiliated Sixth People's Hospital, Shanghai Sixth People's Hospital East Branch and International Peace Maternal and Child Health Hospital of the China Welfare Institute, respectively. The inclusion criteria were as follows: 1) woman of Han ethnicity aged 40-60 years; 2) urban residents with registered permanent rights in Shanghai or residence in Shanghai for $>5$ years; 3 ) no severe concomitant diseases involving the heart, liver, kidney, or other vital organs; 4) no previous sleep disorders or symptoms of depression; and 5) clear consciousness with the ability to respond to the questionnaire correctly and fluently and willingness to collaborate and complete the questionnaire. A woman was excluded from the present study if she: 1) was pregnant; 2) was within 1 year postpartum or currently breastfeeding; 3) had a history of psychiatric disorders, a family history of psychiatric disorders, or taken psychotropic drugs in the last 6 months; 4) had received estrogen supplementation therapy in the last 6 months; 5) had taken contraceptive drugs within the last 6 months; or 6) had consumed alcohol or smoked in the last 6 months. Informed consent was obtained from all participants before the start of the study.

In accordance with the inclusion and exclusion criteria, the study sample eventually comprised 1,004 women at A Hospital. Validation sample 1 comprised 440 women at B Hospital, while validation sample 2 comprised 247 women at $\mathrm{C}$ Hospital. The same inclusion and exclusion criteria were applied in the selection of participants from the 3 centers. The study was approved by the Ethics Committee of Shanghai Sixth People's Hospital (2016-R07).

\section{Material and measurements Collection of basic information}

The basic information included basic sociodemographic information and anthropometric measurements obtained using a questionnaire-based survey. The survey was administered by specially trained personnel in a face-to-face manner. The survey included 20 questions that covered each respondent's age, height, weight, educational attainment, marital status, obstetric history, occupational status, chronic diseases and cancer history, menstrual history, menopausal status, and menopause stage. ${ }^{21}$

\section{Evaluation of depressive symptoms}

Depressive symptoms were assessed using a self-rating depression scale (SDS). ${ }^{22}$ The scale consisted of 20 self-rating items. Each item was graded on a scale of 1 to 4 according to 
degree of severity. The total SDS score was $20-80$ points. A person with a total score $\geq 50$ points was defined as having depressive symptoms. ${ }^{23} \mathrm{~A}$ higher score corresponded to more severe depressive symptoms. The SDS has been shown to have adequate reliability for different ethnic groups and populations. $^{24,25}$

\section{Assessment of hot flashes and sweating}

Symptom severity was examined using the Kupperman Menopause Index. ${ }^{26,27}$ Specifically, the frequency of hot flashes and sweating was objectively assessed and assigned a score of 1 to 4: 0 points, the respondent never experienced hot flashes and sweating; 1 point, the respondent had been experiencing hot flashes and sweating $<3$ times per day; 2 points, the respondent had been experiencing hot flashes and sweating 3-9 times per day; and 3 points, the respondent had been experiencing hot flashes and sweating $\geq 10$ times per day.

\section{Assessment of menopausal status}

The menopausal status was evaluated based on the menstrual bleeding pattern in the past 12 months and classified as follows: 1) premenopausal, lack of changes in the regularity of menstrual cycles in the past 12 months; 2) perimenopausal, the presence of 2 or more menstrual cycles with a variance of $>7$ days in cycle length in the adjacent 2 and amenorrhea interval $\geq 60$ days; and 3 ) postmenopausal, the absence of menstrual periods in the past 12 months. The classification is mainly based on the Stages of Reproductive Aging Workshop +10 staging system. ${ }^{28}$

\section{Statistical analysis}

The statistical analysis was performed using SPSS Version 20.0 for Windows (SPSS, Chicago, IL, USA). The normality of the measurement data was examined using histograms, quantile-quantile plots, skewness and kurtosis values, and the Kolmogorov-Smirnov test $(n>50)$. Normally distributed variables are expressed as mean $\pm \mathrm{SD}$. In the cases of a normal distribution and homogeneous variance, statistical comparisons between the groups were performed using analysis of variance. Otherwise, the Kruskal-Wallis rank sum test was used. Non-normally distributed variables are presented as median and interquartile range. Categorical variables are expressed as frequencies (percentage). Rates were compared using the chi-squared test or Kruskal-Wallis rank sum test. Candidate risk factors were preliminarily screened out from the basic demographic data and the factors contributing to hot flashes and sweating using the logistic regression model of the forward stepwise likelihood method. The candidate risk factors were subjected to stratified analysis. Subsequently, a risk scoring system was established by multiplying the $\beta$ coefficients of the statistically significant variables in the model by 10 and then rounding the results to the nearest integers. The degree of similarity between the values predicted by the multivariate model of the Hosmer-Lemeshow test and the observed prevalence rate were determined. $p$-values $<0.05$ indicated a statistically significant difference. The simplified risk scoring system was validated in validation samples 1 and 2. The receiver operating characteristic (ROC) curves were established by plotting sensitivity against (1-specificity) for different cut-offs, and the optimal cut-off point was identified for each ROC curve: the point at which the sum of sensitivity and specificity minus 1 (sensitivity + specificity -1 ) was maximum, namely, the Youden index. The difference between the ROC curve of the study sample and the ROC curves of validation samples 1 and 2 was examined using an $\mathrm{R}$ algorithm. $p$-values $<0.05$ indicated significant differences between the ROC curves.

\section{Results}

A total of 1,850 women were recruited from the 3 centers. Among the 1,850 women, 83 were excluded because they were $<40$ or $>60$ years, took estrogen supplements or psychotropic drugs in the last 6 months, had a history of smoking tobacco or drinking alcohol, or were lactating. An additional 76 patients were also excluded due to incomplete survey data. Therefore, a total of 1,691 women were eventually included in the present study: 1,004 in the study sample, 440 in validation sample 1, and 247 in validation sample 2. Of the 1,004 women in the study sample, 135 had SDS scores $\geq 50$ points. Therefore, the prevalence of depressive symptoms was $13.4 \%$. The prevalence of depressive symptoms was $11.4 \%$ and $13.4 \%$ in validation samples 1 and 2, respectively (Figure 1).

Of the 1,691 women included in the present study, $218 \mathrm{had}$ SDS scores $\geq 50$ points, resulting in a prevalence of $12.9 \%$. Compared with the prevalence of depressive symptoms in validation sample $2(13.4 \%)$ and the study sample (13.4\%), validation sample $1(11.4 \%)$ was low, but the difference was not statistically significant $\left(\chi^{2}=1.238, p=0.538\right)$.

Regarding the frequency of hot flashes and sweating, 487 women in the study sample (48.5\%) had scores $\geq 1$ (ie, had been experiencing hot flashes and sweating $\geq 1-3$ times a day). The frequency of hot flashes and sweating differed significantly among the study sample, validation sample 1 $(47.5 \%)$, and validation sample $2(61.9 \%)\left(\chi^{2}=16.061\right.$, $p=0.000$ ). Regarding menopausal status, perimenopausal women and postmenopausal women accounted for $35.5 \%$ 


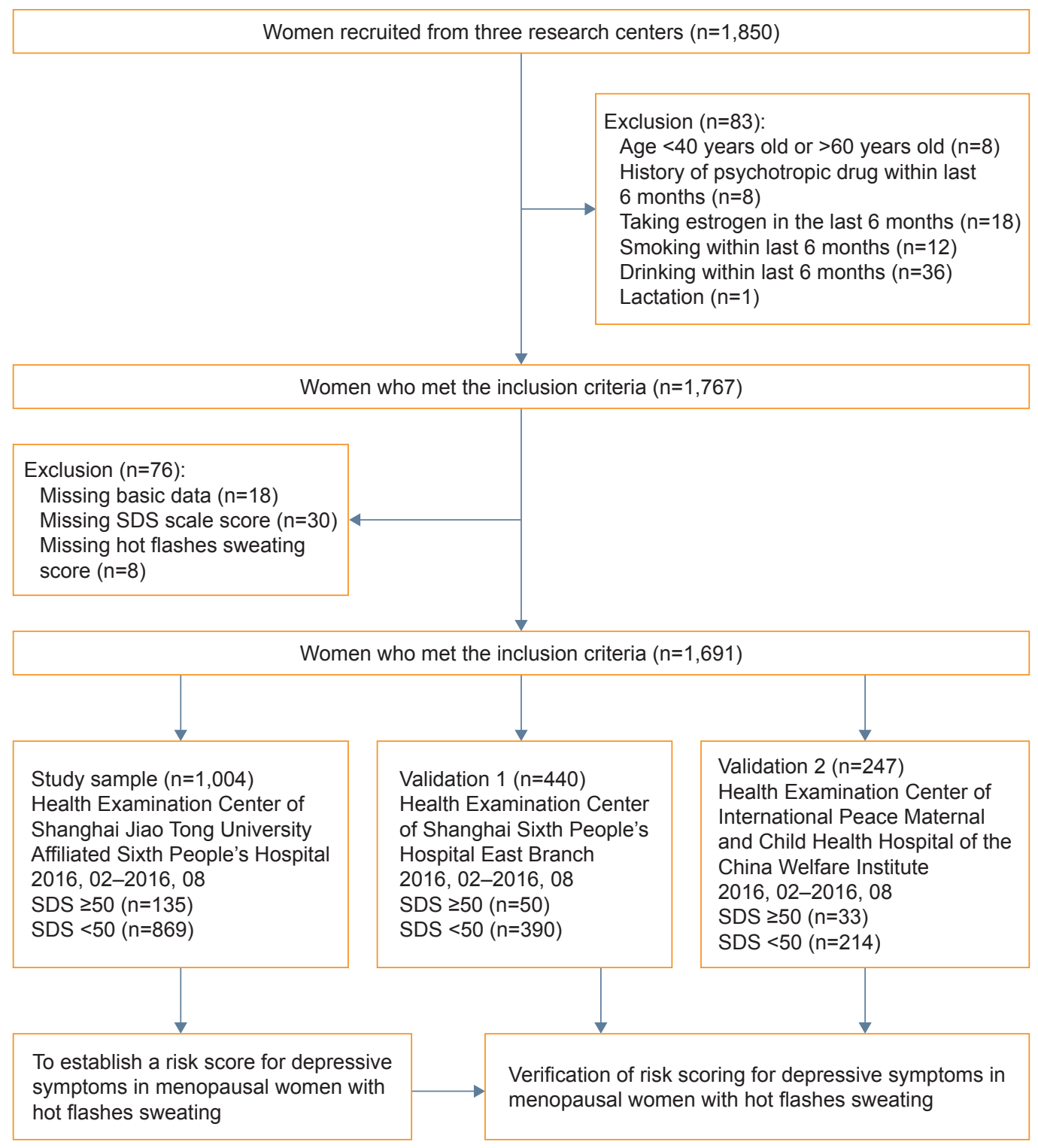

Figure I Process flow diagram.

Abbreviation: SDS, self-rating depression scale.

and $45.8 \%$ of the study sample, $38.0 \%$ and $46.6 \%$ of validation sample 1 , and $40.5 \%$ and $42.5 \%$ of validation sample 2 . Chi-squared tests indicated no significant difference in menopausal status among the 3 samples $\left(\chi^{2}=4.101, p=0.392\right)$. Statistically significant differences existed in body mass index, age at menarche, educational attainment (high school education and below), monthly income ( $\geq 5,000$ yuan), occupational status (employed), nulliparity, surgical menopause, chemotherapy-induced menopause, diabetic status, and hypertensive status among the women from the 3 centers $(p<0.05)$. Further logistic regression analysis using the forward stepwise likelihood method indicated that the occurrence of depressive symptoms was only related to 2 basic demographics characteristics: educational background (below university level) and being diabetic. In contrast, there were no significant differences among the women from the 3 centers in terms of age, marital status (married), hysterectomy-induced menopause, or history of cancer $(p>0.05)$ (Table 1).

The basic information about the study sample and the corresponding scores for hot flashes and sweating were incorporated into the binary logistic regression model to screen for factors influencing the occurrence of depressive symptoms. Depressive symptoms (SDS scores $\geq 50$ points) 
Table I Comparison of the basic characteristics of the women from the three centers

\begin{tabular}{|c|c|c|c|c|}
\hline Characteristics & $\begin{array}{l}\text { Study } \\
\text { sample }\end{array}$ & $\begin{array}{l}\text { Validation } \\
\text { sample I }\end{array}$ & $\begin{array}{l}\text { Validation } \\
\text { sample } 2\end{array}$ & $p$-value \\
\hline Number & $\mathrm{I}, 004$ & 440 & 247 & \\
\hline Age (years) & $50(46-55)$ & $51(46-55)$ & $50(46-54)$ & $0.263^{\mathrm{a}}$ \\
\hline $40-50$ & $508(50.6)$ & $212(48.2)$ & $|3|(53.0)$ & \\
\hline $51-60$ & $496(49.4)$ & $228(5 \mid .8)$ & $116(47.0)$ & \\
\hline BMI $\left(\mathrm{kg} / \mathrm{m}^{2}\right)$ & $23.15 \pm 2.78$ & $23.69 \pm 2.70$ & $22.70 \pm 2.77$ & $0.000^{\mathrm{b}}$ \\
\hline$\geq 0.000$ & $762(75.9)$ & $308(70.0)$ & $20 I(8 I .4)$ & \\
\hline$<25.0$ & $242(24.1)$ & $132(30.0)$ & $46(18.6)$ & \\
\hline Age at menarche (years) & $14.42 \pm 1.72$ & $|4.4| \pm I .56$ & $14.07 \pm 1.30$ & $0.029^{a}$ \\
\hline Married & 991 (98.7) & $431(98.0)$ & $239(96.8)$ & $0.103^{c}$ \\
\hline Employed & $740(73.7)$ & $286(65.0)$ & $187(75.7)$ & $0.00 I^{c}$ \\
\hline Educational attainment (below university level) & $607(60.5)$ & $399(90.7)$ & I7I (69.2) & $0.000^{c}$ \\
\hline Monthly income ( $\geq 5,000$ Yuan) & $425(42.3)$ & $73(16.6)$ & $100(40.5)$ & $0.000^{c}$ \\
\hline Nulliparous & $3 I(3.1)$ & $0(0)$ & $7(2.8)$ & $0.000^{c}$ \\
\hline \multicolumn{5}{|l|}{ Menopausal status } \\
\hline Premenopausal & $188(18.7)$ & $68(15.5)$ & $42(17.0)$ & $0.392^{c}$ \\
\hline Perimenopausal & $356(35.5)$ & $167(38.0)$ & $100(40.5)$ & \\
\hline Postmenopausal & $460(45.8)$ & $205(46.6)$ & $105(42.5)$ & \\
\hline Surgical menopause & $8(0.8)$ & $I(0.2)$ & $9(3.6)$ & $0.000^{c}$ \\
\hline Hysterectomy-induced menopause & $36(3.6)$ & $25(5.7)$ & $13(5.3)$ & $0.153^{c}$ \\
\hline Chemotherapy-induced menopause & $0(0)$ & $4(0.9)$ & I (0.4) & $0.013^{c}$ \\
\hline Hypertensive & $123(12.3)$ & $80(18.2)$ & $26(10.5)$ & $0.003^{c}$ \\
\hline Diabetic & $22(2.2)$ & $25(5.7)$ & $9(3.6)$ & $0.003^{c}$ \\
\hline History of cancer & $15(1.5)$ & $10(2.3)$ & $9(3.6)$ & $0.088^{c}$ \\
\hline \multicolumn{5}{|l|}{ Score for the frequency of hot flashes and sweating } \\
\hline 0 & $517(5 \mid .5)$ & $23 \mid(52.5)$ & $94(38.1)$ & $0.000^{c}$ \\
\hline 1 & $362(36.1)$ & |3| (29.8) & $85(34.4)$ & \\
\hline 2 & $88(8.8)$ & $68(15.5)$ & $54(21.9)$ & \\
\hline 3 & $37(3.7)$ & $10(2.3)$ & $14(5.7)$ & \\
\hline SDS score ( $\geq 50$ points) & $135(13.4)$ & $50(11.4)$ & $33(13.4)$ & $0.538^{c}$ \\
\hline
\end{tabular}

Notes: ${ }^{\mathrm{K}}$ Kruskal-Wallis test for independent samples; ${ }^{\mathrm{b}} \mathrm{One}$-way analysis of variance; ${ }^{\mathrm{c}} \chi{ }^{2}$ test.

Abbreviation: SDS, self-rating depression scale.

were related to menopausal status, scores for hot flashes and sweating, education level (below university level), and diabetic status (being diabetic).

The Hosmer-Lemeshow test showed that the predicted prevalence of depressive symptoms matched the prevalence of depressive symptoms obtained in the actual survey $\left(\chi^{2}=6.714, p=0.243\right)$. A risk scoring system was established based on the logistic regression model (Table 2). The scoring system generated a total score of $0-54$ points, and the optimal cut-off score to screen for depressive symptoms in the women participating in the present study was 31 points. Among the 1,004 women in the study sample, 204 (20.3\%) scored at least 31 points. Based on the SDS screening results, 135 of the 1,004 women (13.4\%) suffered from depressive symptoms (SDS score $\geq 50$ points). Among the 440 women in validation sample $1,129(29.3 \%)$ scored at least 31 points. SDS screening indicated that 50 of the 440 women (11.4\%)
Table 2 Analysis of depressive symptom risk scores of the I,004 subjects using the odds ratio $(\mathrm{OR}, 95 \% \mathrm{Cl})$ and $\beta$ coefficient of the logistic regression model

\begin{tabular}{|c|c|c|c|}
\hline Influence factors & $\beta$ coefficient & OR (95\% CI) & Score \\
\hline \multicolumn{4}{|l|}{ Menopausal status } \\
\hline Premenopausal & - & I & 0 \\
\hline Perimenopausal & 1.585 & $4.88(1.70-14.06)$ & 16 \\
\hline Postmenopausal & 1.25 & $3.49(1.21-10.10)$ & 13 \\
\hline \multicolumn{4}{|c|}{ Score for hot flashes and sweating } \\
\hline 0 & - & I & 0 \\
\hline I & 0.883 & $2.42(1.5 I-3.87)$ & 9 \\
\hline 2 & 1.682 & $5.37(2.93-9.86)$ & 17 \\
\hline 3 & 1.822 & $6.19(2.79-13.70)$ & 18 \\
\hline \multicolumn{4}{|c|}{ Educational level (below university level) } \\
\hline No & - & I & 0 \\
\hline Yes & 0.873 & $2.39(1.51-3.80)$ & 9 \\
\hline \multicolumn{4}{|l|}{ History of diabetes } \\
\hline No & - & I & 0 \\
\hline Yes & 1.12 & 3.07 (1.19-7.91) & II \\
\hline
\end{tabular}

Abbreviation: OR, odds ratio. 
developed depressive symptoms (SDS score $\geq 50$ points). Among the 247 women in validation sample 2, 84 (34.1\%) scored at least 31 points. SDS screening indicated that 33 of the 247 women (13.4\%) developed depressive symptoms (SDS score $\geq 50$ points). The area under the ROC curve of the study sample was 0.750 (95\% CI, 0.708-0.793). The risk score of validation sample 1 had an area under the curve (AUC) of 0.731 (95\% CI, 0.667-0.794), while the risk score of validation sample 2 had an AUC of 0.744 (95\% CI, 0.669-0.820) (Figure 2). At the optimal cuff-off score of $\geq 31$ points, the ROC curve of the study sample showed that the sensitivity and specificity were 0.667 and 0.701 , respectively, and the Youden index was 0.357. At the same cuff-off point, the sensitivity and specificity for validation sample 1 were 0.840 and 0.621 , respectively, while the sensitivity and specificity for validation sample 2 were 0.879 and 0.606 , respectively. If the selected optimal cut-off score was $\geq 28$ points, the sensitivity was changed to 0.860 and 0.879 for validation sample 1 and validation sample 2, respectively, while the specificity was changed to 0.612 and 0.573 , respectively. Sensitivity and specificity both displayed stable changes. Calculation using $\mathrm{R}$ showed
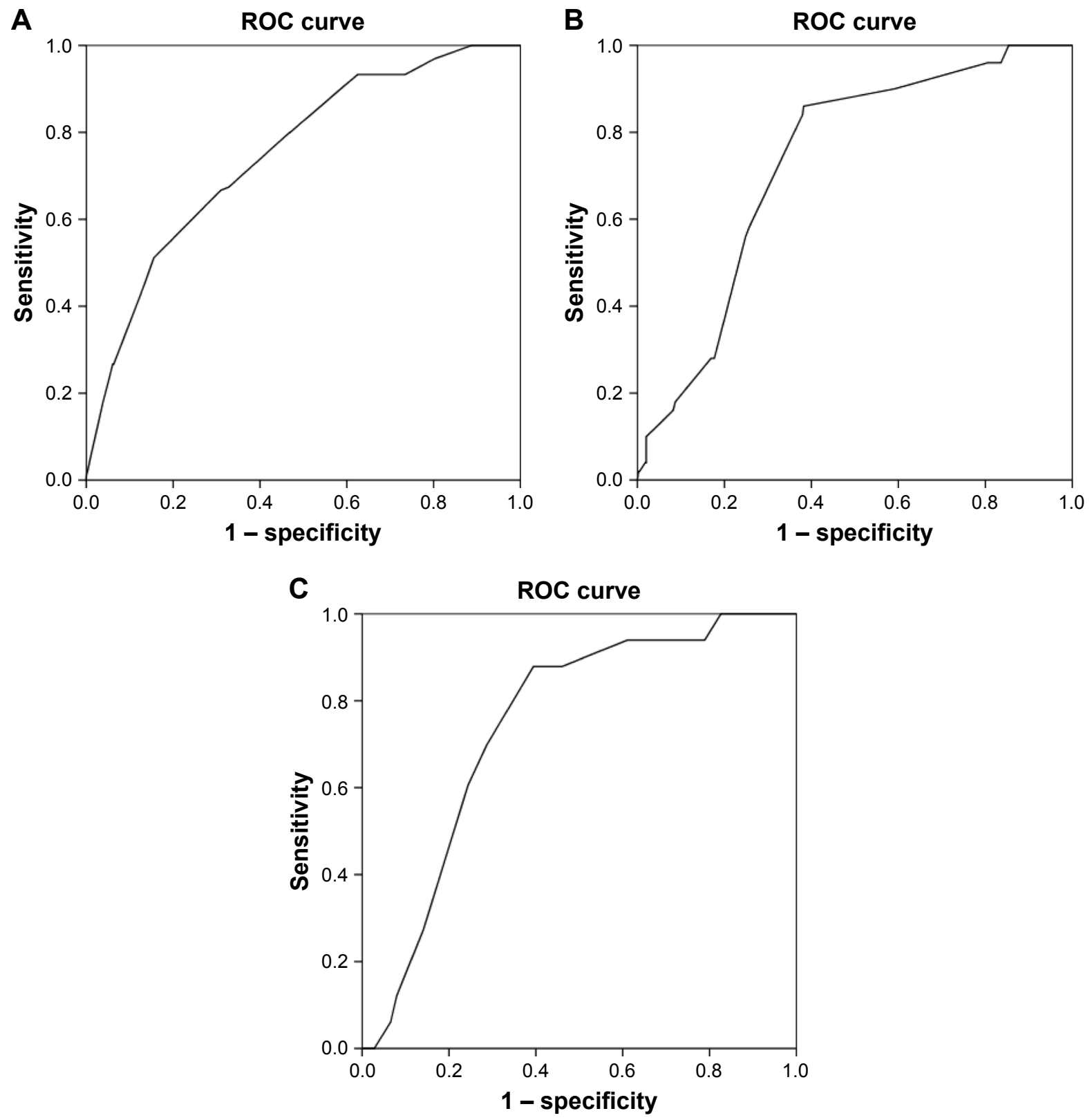

Figure 2 ROC curves for depressive symptom risk scores.

Notes: Risk scores for prediction of the occurrence of depressive symptoms. (A) Study sample; (B) validation sample I; (C) validation sample 2.

Abbreviation: ROC, receiver operating characteristic. 
no significant difference in AUC between the study sample and validation sample $1(p=0.6162)$ and no significant difference in AUC between the study sample and validation sample $2(p=0.8958)$.

\section{Discussion}

In the present multicenter study, a model for predicting the risk of depressive symptoms in menopausal women was established based on hot flashes and sweating. In addition, the model was validated by analyzing the survey data from 2 other centers. To our knowledge, our risk prediction model for depressive symptoms in menopausal women is the first to be established based on hot flash and sweating symptoms. Our model is more convenient for predicting the risk of depressive symptoms.

Many studies have reported that the major factors influencing the occurrence of depressive symptoms in menopausal women include menopause, hot flashes, sweating, and endocrine factors. ${ }^{15,16,29}$ Gibson et al reported that depressive symptoms are more likely to occur in women at early perimenopause than in women at late perimenopause. ${ }^{15}$ Berent-Spillson et al reported that endocrine and metabolic factors play a significant role in enhancing the risk of depressive symptoms in menopausal women. ${ }^{29}$ Joffe et al employed a gonadotropin-releasing hormone agonist-based experimental model, compared the experimental groups with the control groups, and found that the depressive symptoms in menopausal women were affected by hot flashes, sweating, and night-time sleep interruption. ${ }^{16}$ However, these studies failed to employ a quantitative index to compare the risks of depressive symptoms at different menopausal stages; also, they did not analyze and predict the risk ratio for important factors such as hot flashes and sweating.

The present study found that menopausal status, frequency of hot flashes and sweating, education level, and diabetes were the main factors influencing the occurrence of depressive symptoms in 40-60-year-old women. Among the previous factors, perimenopause and a frequency of hot flashes and sweating $\geq 10$ times a day had the greatest impact on the occurrence of depressive symptoms in menopausal women. Further model establishment and a comparative study found that the risk ratio was different for each influencing factor. Regarding menopausal status, perimenopause had a greater influence than postmenopause (risk scores, 16:13). Increased severity of hot flashes and sweating enhanced the risk of developing depressive symptoms. The risk scores were as follows: hot flashes and sweating $\geq 10$ times/day, 18 ; hot flashes and sweating 3-9 times/day, 17; hot flashes and sweating $<3$ times/day, 9; no hot flashes and sweating, 0 ; below university education level, 9 ; university education and above, 0 ; with diabetes, 11; and without diabetes, 0 .

The quantitative index in the established scoring system can be used to easily and quickly assess the risk of depressive symptoms. The optimal cut-off score in our model was 31 points. A score $\geq 31$ points indicates a high risk of developing depressive symptoms. Our model allows prediction of the risk of depressive symptoms without laboratory tests, which is conducive to its use in large-scale screening efforts. Moreover, patients should be able to comprehend and use the scoring model. Using this method, a patient can obtain the corresponding score by performing a simple classification of her own frequency of hot flashes and sweating.

The risk score of the study sample had an AUC of 0.750 and sensitivity of 0.667 . Both values were within the acceptable range. In addition, the model was validated using samples collected from 2 other centers (AUC values of 0.731 and 0.744 and sensitivities of 0.840 and 0.879 ). Calculation using $\mathrm{R}$ revealed no difference in AUC values between the validation samples and the study sample, indicating the model's accuracy.

The present study found that the prevalence of depressive symptoms in women aged 40-60 years was $13.4 \%$ (the study sample), $11.4 \%$ (validation sample 1), and 13.4\% (validation sample 2). The overall prevalence was $12.9 \%$ (total of 1,691 women), which was lower than the prevalence $(23.8 \%)$ reported by foreign SWAN researchers while not significantly different from the prevalence (11.4\%) reported by Chinese scholars. ${ }^{30-32}$ The results of the present study also showed that the prevalences of hot flashes and sweating in women aged 40-60 years (score for hot flashes and sweating $\geq 1$ point, ie, the women experienced hot flashes and sweating at least 1-3 times a day) were $48.5 \%$ (the study sample), $47.5 \%$ (validation sample 1), and 61.9\% (validation sample 2). The overall prevalence was 50.2\% (total of 1,691 women), which was lower than the prevalence reported by Nie et al $(80.7 \%)$ and consistent with the results of our previous study. ${ }^{33}$

Hot flashes and sweating are factors that influence depressive symptoms in menopausal women. Studies also show that depressive symptoms impact and even aggravate the severity of hot flashes and sweating. ${ }^{34,35}$ Therefore, there may be a mutual influence and reciprocal causal relation between menopausal symptoms due to postmenopausal hormonal changes on multiple organs. Hot flashes and sweating through some means affect the daily life and personal subjective feeling. ${ }^{36}$ Studies have shown that hot sweating on the 
impact of depressive symptoms is even more significant when combined with social factors (such as education). The reason maybe is different levels of education of people with different knowledge awareness, different self-learning ability, affecting the personal emotional guidance. Diabetes mellitus is one of the most common chronic diseases in women after menopause. Patients experience a number of co-morbidities, including physical and psychological problems. ${ }^{37}$ Depression is co-morbid, modifiable psychological condition associated with diabetes. Peri-menopause women generally have irregular menstrual cycle. Increased abdominal fat caused by ovarian failure may lead to insulin sensitivity and glucose metabolic disorders in postmenopausal women. ${ }^{38}$ Changes in menopause and postmenopausal hormone levels can cause fluctuations in blood glucose levels. ${ }^{39}$ Changes in hormone levels may also lead to weight gain.

The principles and underlying mechanisms are rather complex and require further exploration. Reports in the literature indicate that nationality, education level, menopausal status, exercise, sleep apnoea, and cessation of hormone replacement therapy (HRT) are also risk factors for a high incidence of depressive symptoms in menopausal women. ${ }^{1,40-42}$ Menopausal status and education level correspond to the influencing factors identified in the present study. Due to the complex diversity of the influencing factors, the present study did not examine different ethnic groups, sleep status, or HRT use. Therefore, incorporating additional influential factors and including many laboratory tests will be the focus of our next study. In addition, previous reports suggested that the development of depressive symptoms in menopausal women would increase the risk of chronic and cardiovascular diseases. Therefore, further expansion of the study scope will also be the focus of further studies. ${ }^{43,44}$ On the other hand, we have to do more and more in the treatment of menopausal symptoms, including depression symptoms. Some researchers think dietary supplements can also improve the quality of life of menopausal women and reduce symptoms associated with menopause, including depressive symptoms. ${ }^{45-48}$ Maybe this is a nice way outside of HRT.

\section{Conclusion}

A scoring system for predicting the risk of depressive symptoms in menopausal women based on hot flash and sweating symptoms was established in this multicenter study. The risk scoring system was validated using small samples from 2 other centers. Due to China's large population, further studies should expand the sample size and perform validation analysis using samples from additional research centers. The risk scoring system established in the present study is simple and convenient to use. The score provides a simple screening tool capable of replacing the existing complex assessment scales and is suitable for use in initial and large-scale screenings.

\section{Acknowledgments}

The authors thank Shanghai Municipal Science and Technology Commission (grant 15211950202) and Shanghai Municipal Commission of Health and Family Planning (grant 201440004 and GWIV-15), for providing funding for this study. The authors also acknowledge the Health Examination Center of Shanghai JiaoTong University Affiliated Sixth People's Hospital, the Health Examination Center of Shanghai Sixth People's Hospital East Branch and the Health Examination Center of International Peace Maternal and Child Health Hospital of the China Welfare Institute.

\section{Disclosure}

The authors report no conflicts of interest in this work.

\section{References}

1. Hickey M, Schoenaker DA, Joffe H, Mishra GD. Depressive symptoms across the menopause transition: findings from a large population-based cohort study. Menopause. 2016;23(12):1287-1293.

2. Santoro N, Epperson CN, Mathews SB. Menopausal symptoms and their management. Endocrinol Metab Clin North Am. 2015;44(3):497-515.

3. Campbell KE, Dennerstein L, Finch S, Szoeke CE. Impact of menopausal status on negative mood and depressive symptoms in a longitudinal sample spanning 20 years. Menopause. 2016;24(5):490-496.

4. de Kruif M, Spijker AT, Molendijk ML. Depression during the perimenopause: a meta-analysis. J Affect Disord. 2016;206:174-180.

5. Cohen LS, Soares CN, Vitonis AF, Otto MW, Harlow BL. Risk for new onset of depression during the menopausal transition: the Harvard study of moods and cycles. Arch Gen Psychiatry. 2006;63(4):385-390.

6. Bromberger JT, Matthews KA, Schott LL, et al. Depressive symptoms during the menopausal transition: the Study of Women's Health Across the Nation (SWAN). J Affect Disord. 2007;103(1-3):267-272.

7. Bromberger JT, Kravitz HM. Mood and menopause: findings from the Study of Women's Health Across the Nation (SWAN) over 10 years. Obstet Gynecol Clin North Am. 2011;38(3):609-625.

8. Soares CN. Mood disorders in midlife women: understanding the critical window and its clinical implications. Menopause. 2014;21(2): 198-206.

9. Rodríguez MR, Nuevo R, Chatterji S, Ayuso-Mateos JL. Definitions and factors associated with subthreshold depressive conditions: a systematic review. BMC Psychiatry. 2012;12(1):300-305.

10. Rössler W, Ajdacic-Gross V, Riecher-Rössler A, Angst J, Hengartner MP. Does menopausal transition really influence mental health? Findings from the prospective long-term Zurich study. World Psychiatry. 2016; 15(2):146-154.

11. Chuni N, Sreeramareddy CT. Frequency of symptoms, determinants of severe symptoms, validity of and cut-off score for Menopause Rating Scale (MRS) as a screening tool: a cross-sectional survey among midlife Nepalese women. BMC Womens Health. 2011;11:30.

12. Fisher WI, Thurston RC. Measuring hot flash phenomenonology using ambulatory prospective digital diaries. Menopause. 2016;23(11): 1222-1227.

13. Huicochea-Gómez L, Sievert LL, Cahuich-Campos D, Brown DE. An investigation of life circumstances associated with the experience of hot flashes in Campeche, Mexico. Menopause. 2017;24(1):52-63. 
14. Santoro N, Epperson CN, Mathews SB. Menopausal symptoms and their management. Endocrinol Metab Clin North Am. 2015; 44(3):497-515.

15. Gibson CJ, Joffe H, Bromberger JT, et al. Mood symptoms after natural menopause and hysterectomy with and without bilateral oophorectomy among women in midlife. Obstet Gynecol. 2012;119(5):935-941.

16. Joffe H, Crawford SL, Freeman MP, et al. Independent contributions of nocturnal hot flashes and sleep disturbance to depression in EstrogenDeprived Women. J Clin Endocrinol Metab. 2016;101(10):3847-3855.

17. Joffe H, Soares CN, Cohen LS. Assessment and treatment of hot flushes and menopausal mood disturbance. Psychiatric Clin North Am. 2003;26(3):563-580.

18. Ladd CO, Newport DJ, Ragan KA, Loughhead A, Stowe ZN. Venlafaxine in the treatment of depressive and vasomotor symptoms in women with perimenopausal depression. Depress Anxiety. 2005; 22(2):94-97.

19. Zhou X, Qiao Q, Ji L, et al. Nonlaboratory-based risk assessment algorithm for undiagnosed type 2 diabetes developed on a nation-wide diabetes survey. Diabetes Care. 2013;36(12):3944-3952.

20. Nygaard RM, Sirany AM, Wyman EA, et al. A Clinical tool to risk stratify potential kidney transplant recipients and predict severe adverse events. Clin Transplant. 2016;30(11):1494-1500.

21. Sun D, Shao H, Li C, Tao M. Sleep disturbance and correlates in menopausal women in Shanghai. J Psychosom Res. 2014;76(3):237-241.

22. Romera I, Delgado-Cohen H, Perez T, Caballero L, Gilaberte I. Factor analysis of the Zung self-rating depression scale in a large sample of patients with major depressive disorder in primary care. $B M C$ Psychiatry. 2008;8(1):4.

23. Wu Y, Si R, Yang S, et al. Depression induces poor prognosis associates with the down-regulation brain derived neurotrophic factor of serum in advanced small cell lung cancer. Oncotarget. 2016;7(52):85975-85986.

24. Xia Y, Wang N, Yu B, et al. Dietary patterns are associated with depressive symptoms among Chinese adults: a case-control study with propensity score matching. Eur J Nutr. Epub 2016 Aug 19.

25. Sharpley CF, Christie DR, Bitsika V, Miller BJ. Trajectories of total depression and depressive symptoms in prostate cancer patients receiving six months of hormone therapy. Psychooncology. 2017;26(1):60-66.

26. Tao MF, Shao HF, Li CB, Teng Y. Correlation between the modified Kupperman Index and the Menopause Rating Scale in Chinese women. Patient Prefer Adherence. 2013;7(3):223-229.

27. Kalay AE, Demir B, Haberal A, Kandemir O. Efficacy of citalopram on climacteric symptoms. Menopause. 2007;14(2):223-229.

28. Harlow SD, Gass M, Hall JE, et al. Executive summary of the Stages of Reproductive Aging Workshop +10: addressing the unfinished agenda of staging reproductive aging. Menopause. 2012;19(4):387-395.

29. Berent-Spillson A, Marsh C, Persad C, Randolph J, Zubieta JK, Smith Y. Metabolic and hormone influences on emotion processing during menopause. Psychoneuroendocrinology. 2017;76:218-225.

30. Gibson CJ, Thurston RC, Matthews KA. Cortisol dysregulation is associated with daily diary-reported hot flashes among midlife women. Clin Endocrinol (Oxf). 2016;85(4):645-651.

31. Zang H, He L, Chen Y, Ge J, Yao Y. The association of depression status with menopause symptoms among rural midlife women in China. Afr Health Sci. 2016;16(1):97-104.
32. Kopciuch D, Paczkowska A, Zaprutko T, Michalak M, Nowakowska E Conventional or natural pharmacotherapy and the prevalence of anxiety and depression during menopause. Altern Ther Health Med. 2017 ; 23(2):12-19.

33. Nie G, Yang H, Liu J, Zhao C, Wang X. Psychometric properties of the Chinese version of the Menopause-Specific Quality-of-Life questionnaire. Menopause. 2017;24(5):546-554.

34. Melby MK, Anderson DJ, Sievert LL, Obermeyer CM. Methods used in cross-cultural comparisons of vasomotor symptoms and their determinants. Maturitas. 2011;70(2):110-119.

35. Singhal SR, Shullai WK. Comparative study of gabapentin and isoflavone in menopausal vasomotor symptoms. $J$ Midlife Health. 2016;7(3):132-139.

36. Brown L, Bryant C, Brown VM, Bei B, Judd FK. Self-compassion weakens the association between hot flushes and night sweats and daily life functioning and depression. Maturitas. 2014;78(4):298-303.

37. Hasan SS, Thiruchelvam K, Ahmed SI, Clavarino AM, Mamun AA, Kairuz T. Pregnancy complications, mental health-related problems and type 2 diabetes mellitus in Malaysian women. Diabetes Metab Syndr. 2013;7(4):191-197.

38. Szmuilowicz ED, Stuenkel CA, Seely EW. Influence of menopause on diabetes and diabetes risk. Nat Rev Endocrinol. 2009;5(10):553-558.

39. Otsuki M, Kasayama S, Morita S, et al. Menopause, but not age, is an independent risk factor for fasting plasma glucose levels in non-diabetic women. Menopause. 2007;14(3 Pt 1):404-407.

40. Kai Y, Nagamatsu T, Kitabatake Y, Sensui H. Effects of stretching on menopausal and depressive symptoms in middle-aged women: a randomized controlled trial. Menopause. 2016;23(8):827-832.

41. Perich TA, Roberts G, Frankland A, et al. Clinical characteristics of women with reproductive cycle-associated bipolar disorder symptoms. Aust N Z J Psychiatry. 2017;51(2):161-167.

42. Shors TJ, Leuner B. Estrogen-mediated effects on depression and memory formation in females. J Affect Disord. 2003;74(1):85-96.

43. Hui LY, Wang YW, Zhou FL, et al. Association between MKP-1, BDNF, and Gonadal Hormones with depression on perimenopausal women. J Womens Health (Larchrnt). 2016;25(1):71-77.

44. Bromberger JT, Kravitz HM, Chang YF, Cyranowski JM, Brown C, Matthews KA. Major depression during and after the menopausal transition: Study of Women's Health Across the Nation (SWAN). Psychol Med. 2011;41(9):1879-1888.

45. Davinelli S, Scapagnini G, Marzatico F, Nobile V, Ferrara N, Corbi G. Influence of equol and resveratrol supplementation on health-related quality of life in menopausal women: a randomized, placebo-controlled study. Maturitas. 2017;96:77-83.

46. Lipovac M, Chedraui P, Gruenhut C, et al. The effect of red clover isoflavone supplementation over vasomotor and menopausal symptoms in postmenopausal women. Gynecol Endocrinol. 2012;28(3):203-207.

47. Carmignani LO, Pedro AO, Costa-Paiva LH, Pinto-Neto AM. The effect of dietary soy supplementation compared to estrogen and placebo on menopausal symptoms: a randomized controlled trial. Maturitas. 2010;67(3):262-269.

48. Scapagnini G, Davinelli S, Drago F, De Lorenzo A, Oriani G. Antioxidants as antidepressants: fact or fiction? CNS Drugs. 2012;26(6): $477-490$.
Clinical Interventions in Aging

\section{Publish your work in this journal}

Clinical Interventions in Aging is an international, peer-reviewed journal focusing on evidence-based reports on the value or lack thereof of treatments intended to prevent or delay the onset of maladaptive correlates of aging in human beings. This journal is indexed on PubMed Central, MedLine,

\section{Dovepress}

CAS, Scopus and the Elsevier Bibliographic databases. The manuscript management system is completely online and includes a very quick and fair peer-review system, which is all easy to use. Visit http://www.dovepress. com/testimonials.php to read real quotes from published authors. 